\title{
Interim core concepts for a bayesian pure exchange economy
}

\author{
Tatsuro Ichiishi ${ }^{\mathrm{a}, *}$, Akira Yamazaki ${ }^{\mathrm{b}}$ \\ a Department of Economics, Ohio State University, Columbus, OH 43210-1172, USA \\ ${ }^{\mathrm{b}}$ Graduate School of Economics, Hitotsubashi University, Kunitachi, Tokyo 186-8601, Japan
}

Received 26 August 2003; received in revised form 18 September 2003; accepted 7 October 2003

\begin{abstract}
The private information case of a Bayesian pure exchange economy is studied. The Bayesian incentive-compatible coarse core is proved to be nonempty. On the other hand, the Bayesian incentive-compatible interim core may be empty, even for Bayesian pure exchange economies satisfying the traditional (neoclassical) assumptions. A sufficient condition for the latter's nonemptiness is established; the condition specifies relationships among effects of different type profiles. (C) 2003 Elsevier B.V. All rights reserved.
\end{abstract}

Keywords: Bayesian pure exchange economy; Private measurability; (Bayesian) incentive compatibility; Incentive-compatible coarse core; Incentive-compatible interim core

\section{Introduction}

A Bayesian pure exchange economy is a pure exchange economy in which the consumers are asymmetrically endowed with information about their preference relations and initial endowments. A strategy in a Bayesian pure exchange economy is a plan which specifies a net trade for each state. ${ }^{1}$ We adopt Harsanyi's (1967/68) type-profile approach, so a state is defined as a type profile. A strategy bundle is endogenously determined as a solution of the game played by the consumers. A strategy bundle is synonymously called a mechanism, so the theory endogenously determines a mechanism. After the consumers determine a mechanism, they execute it in the interim period, that is, each consumer chooses his particular net trade according to the plan when he knows his own type but not the others'. A solution is

\footnotetext{
* Corresponding author.

E-mail addresses: ichiishi.1@osu.edu (T. Ichiishi), yamazaki@econ.hit-u.ac.jp (A. Yamazaki).

1 Some works define a plan as a function which specifies a consumption bundle for each state, or as a pair of a net-trade plan and a communication plan, but the present paper does not consider these strategies.
} 
called interim if it is determined in the interim period. It is called ex ante if it is determined in the ex ante period.

Wilson (1978) initiated analysis of cooperative behavior in a Bayesian pure exchange economy, and proposed the coarse core and the fine core as interim solution concepts. He established nonemptiness of the coarse core, and also provided an example of an economy with an empty fine core.

Subsequent researchers paid particular attention to two basic conditions that a strategy needs to satisfy, in that the members of a coalition only design a strategy bundle which satisfies these two conditions. One condition, originally proposed by Radner (1968) in a different context, is the informational feasibility. It is the requirement that each plan be measurable with respect to the information structure available to the consumer at the time of his strategy-execution. Yannelis (1991) considered the private information case defined as the situation in which only the private information structure will be available to each consumer at the time of strategy-execution. A strategy bundle satisfying the informational feasibility for the private information case is called private measurable.

Another condition is the requirement that a strategy bundle be exactly executed according to the agreed upon plan, namely d'Aspremont and Gérard-Varet's (1979) Bayesian incentive compatibility. Ichiishi and Idzik (1996) introduced this requirement into a generalized core analysis, and defined the ex ante Bayesian incentive-compatible core as the core in the private information case in which each possible coalition designs a private measurable and Bayesian incentive-compatible strategy bundle in the ex ante period.

Our study object in the present paper is the interim analogues of the ex ante Bayesian incentive-compatible core. We introduce the Bayesian incentive compatibility to Wilson's coarse core and a variant of his fine core in the private information case.

Our first result is the general nonemptiness theorem for the Bayesian incentive-compatible coarse core. This positive result makes a sharp contrast to Vohra's (1999, Example 3.2, pp. 136-138) negative result; the latter is based on the postulate that execution of a coalitional strategy is assisted and enforced by a mediator, so each strategy can utilize pooled information despite the fact that consumers do not share their private information. Our private information case is appropriate in an economy in which no coalition uses a mediator or shares their private information. We view that in general there is no mediator in the present-day economy, so the private information case prevails, unless there is another mechanism built in the economy for information-transmission or information-sharing.

Wilson (1978) defined the fine core for situations in which the members of a coalition can use several communication systems for pooling their private information. In the absence of a credible communication system, however, we are left with the private information case, and the interim core concept which is essentially as fine as Wilson's fine core concept is naturally defined in this case. This is the Bayesian incentive-compatible interim core. Vohra (1999, Example 2.1, pp. 131-132) constructed an example of a market for a single indivisible lemon with money in which the Bayesian incentive-compatible interim core is empty. By removing the indivisibility from his example, we see that this negative result may hold true even for Bayesian pure exchange economies which satisfy all the standard neoclassical assumptions. The Bayesian incentive-compatible interim core may be empty, because a utility allocation is blocked by a coalition at a particular type profile $\left(S, t^{S}\right)$, and another utility allocation which is stable against $\left(S, t^{S}\right)$ is blocked by coalition at another 
type profile $\left(S^{\prime}, t^{\prime S^{\prime}}\right)$. In order to guarantee the existence of a core strategy bundle, therefore, we need to explore the effects of different type profiles on the stability of strategy plans. The second result of the present paper is to establish a condition on the effects of different type profiles under which the Bayesian incentive-compatible interim core is nonempty. However, the second result is based on the strong postulate that each consumer's interim expected utility given his type is affine linear. Moreover, the condition for nonemptiness of the interim core is stringent. Nevertheless, at the cost of these strong assumptions, we have obtained simplicity of the sufficient condition, which offers a clear-cut insight into the relationship among different type profiles that is needed for the positive result.

We remark that the present positive results are unlikely to be extended beyond the pure exchange economy.

For a survey of the works on ex ante cores of a Bayesian pure exchange economy, see, e.g., Ichiishi and Yamazaki (2002, Subsection 3.5).

The next section formulates the model of Bayesian pure exchange economy, and two descriptive solutions: a Bayesian incentive-compatible coarse core net-trade plan, and a Bayesian incentive-compatible interim core net-trade plan. Section 3 presents the main results: the two existence results. Section 4 discusses several examples in order to gain an insight into some aspects of our assumption on effects of type profiles. Section 5 provides proofs of all the results. The Bayesian incentive-compatible interim core concept actually does not take into account the effects of possible revelation of private-information by the very act of agreeing or disagreeing to a coalitional (coordinated) strategy bundle. The last section points out a simple situation in which such revelation does not affect a consumer's decision in regard to joining the coalition.

\section{Model and two interim core concepts}

We study a pure exchange economy with $l$ commodities and $n$ consumers in which consumers are asymmetrically endowed with information about the data of the economy. Let $N$ be the set of $n$ consumers, and let $\mathcal{N}\left(:=2^{N} \backslash\{\emptyset\}\right)$ be the family of nonempty coalitions. In accordance with Harsanyi's (1967/1968) framework of the Bayesian game, let $T^{j}$ be a finite set of consumer $j$ 's types. The type-profile space for each coalition $S$ is then given as $T^{S}:=\prod_{j \in S} T^{j}$. Set for simplicity, $T:=T^{N}$. A member of $T$ is synonymously called a type profile or a state. The interim period is defined here as the period during which each consumer $j$ knows his true type $t^{j} \in T^{j}$ but not the others'. His private information structure, denoted by $\mathcal{T}^{j}$, is the algebra on $T$ generated by the sets, $\left\{t^{j}\right\} \times T^{N \backslash\{j\}}, t^{j} \in T^{j}$.

Given type profile $t$, each consumer is characterized by his consumption set $\mathbf{R}_{+}^{l}$, his statedependent von Neumann-Morgenstern utility function $u^{j}(\cdot, t): \mathbf{R}_{+}^{l} \rightarrow \mathbf{R}$, his initial endowment vector $e^{j}\left(t^{j}\right) \in \mathbf{R}_{+}^{l}$, and his interim probability $\pi^{j}\left(\cdot \mid t^{j}\right)$ on $T$ given $t^{j}$. We are assuming here that the initial endowment function $e^{j}$ depends only on $t^{j}$, in other words, $e^{j}$ (when viewed as a function on $T$ ) is $\mathcal{T}^{j}$-measurable. If the consumers commonly hold an objective ex ante probability $\pi$ on $T$, consumer $j$ 's interim probability is given by the Bayes' rule,

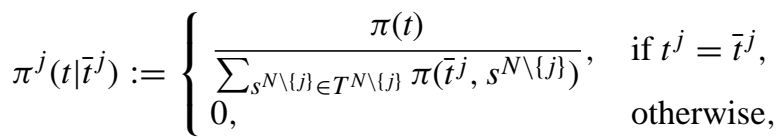


but our present framework allows for subjective probabilities, and even inconsistency among interim probabilities (in that there is no ex ante probability to which the Bayes' rule can be applied in order to derive the interim probabilities).

Definition 2.1. A Bayesian pure exchange economy

$$
\mathcal{E}_{p e}:=\left\{T^{j}, \mathbf{R}_{+}^{l}, u^{j}, e^{j},\left\{\pi^{j}\left(\cdot \mid t^{j}\right)\right\}_{t^{j} \in T^{j}}\right\}_{j \in N}
$$

is an economy with $l$ commodities, where $N$ is a consumer set, and for each consumer $j, T^{j}$ is his type set, $\mathbf{R}_{+}^{l}$ is his consumption set, $u^{j}: \mathbf{R}_{+}^{l} \times T \rightarrow \mathbf{R}$ is his type-profile-dependent von Neumann-Morgenstern utility function, $e^{j}: T \rightarrow \mathbf{R}_{+}^{l}$ is his initial endowment function, postulated to be $\mathcal{T}^{j}$-measurable, and $\pi^{j}\left(\cdot \mid t^{j}\right)$ is his interim probability on the states $T$ given $t^{j}$.

We analyze consumers' cooperative behavior during the interim period. When type profile $t$ prevails, consumer $j$ chooses his net trade vector $z^{j}(t)\left(\in \mathbf{R}^{l}\right)$. Any set $S$ of consumers can come together and jointly schedule a net-trade plan $z^{S}:=\left\{z^{j}\right\}_{j \in S}$, so that the plan is individually feasible,

$$
\forall t \in T: \forall j \in S: z^{j}(t)+e^{j}\left(t^{j}\right) \in \mathbf{R}_{+}^{l},
$$

and is attainable,

$$
\forall t \in T: \sum_{j \in S} z^{j}(t) \leq \mathbf{0}
$$

Consumer $j$ 's net-trade plan $z^{j}: T \rightarrow \mathbf{R}^{l}$ is his strategy. ${ }^{2}$ Denote by $F^{S}$ the set of all feasible strategy bundles for coalition $S$ :

$$
F^{S}:=\left\{z^{S}: T \rightarrow \mathbf{R}^{l \cdot \# S} \mid z^{S} \text { satisfies (1) and (2) }\right\} .
$$

Not all plans in $F^{S}$ can actually be chosen. We discuss two basic conditions that a plan has to satisfy. We are studying consumers' behavior in (what the literature has called) the private information case, that is the private information structure $\mathcal{T}^{j}$ is the information structure available to $j$ at the time of $j$ 's action (choice of net trade). The first condition, therefore, stipulates that a plan be informationally feasible in this case; to be precise,

$$
\forall j \in S: z^{j} \text { is } \mathcal{T}^{j}-\text { measurable. }
$$

A plan $z^{S}$ satisfying condition (3) is called private measurable. Denote by $F^{\prime S}$ the set of all feasible, private-measurable strategy bundles for coalition $S$ :

$$
F^{\prime S}:=\left\{z^{S} \in F^{S} \mid z^{S} \text { satisfies (3) }\right\} .
$$

The second condition pertains to the feasibility of execution of strategy bundles viewed as "contracts" made within a coalition. Suppose that the members of coalition $S$ agree to

\footnotetext{
${ }^{2}$ It is important that $j$ 's strategy is his net-trade plan $z^{j}$. Some models postulate that $j$ 's strategy is his consumption plan, $t \mapsto z^{j}(t)+e^{j}\left(t^{j}\right)$, but we cannot obtain the existence results (the main results of this paper) with this alternative definition of strategy.
} 
take a strategy bundle $z^{S} \in F^{\prime S}$. Let $\bar{t}^{j}$ be consumer $j$ 's true type. If he makes a choice according to the agreement, his interim expected utility given his true type is

$$
E u^{j}\left(z^{j}+e^{j} \mid \bar{t}^{j}\right):=\sum_{t \in T} u^{j}\left(z^{j}\left(\bar{t}^{j}\right)+e^{j}\left(\bar{t}^{j}\right), t\right) \pi^{j}\left(t \mid \bar{t}^{j}\right) .
$$

In the private information case, however, he can make any choice $c^{j} \in z^{j}\left(T^{j}\right) \backslash\left\{z^{j}\left(\bar{t}^{j}\right)\right\}$ contrary to the agreement, yet his colleagues $S \backslash\{j\}$ cannot catch this betraying act, being led to believe that $j$ 's true type were in the event $\left(z^{j}\right)^{-1}\left(c^{j}\right)$. If $j$ makes such a choice, his interim expected utility given his true type is

$$
E u^{j}\left(c^{j}+e^{j} \mid \bar{t}^{j}\right):=\sum_{t \in T} u^{j}\left(c^{j}+e^{j}\left(\bar{t}^{j}\right), t\right) \pi^{j}\left(t \mid \bar{t}^{j}\right) .
$$

The members of the coalition decide on plan $z^{S}$, in order to realize the choice bundle $z^{S}(t)$ at each possible type profile $t$. If member $j$ does not act according to the agreed plan, taking advantage of his private information, the required outcome $z^{S}(\bar{t})$ cannot be achieved and the purpose of the coalition formation is not fulfilled. To guarantee realization of the exact execution of an agreement, the members of the coalition agree only to those plans $z^{S}$ which nobody has an incentive to act contrary to. In short, they agree only to the Bayesian incentive-compatible plans. To be precise, the Bayesian incentive compatibility is defined as:

$$
\forall t^{j}, \tilde{t}^{j} \in T^{j}: E u^{j}\left(z^{j}+e^{j} \mid t^{j}\right) \geq E u^{j}\left(z^{j}\left(\tilde{t}^{j}\right)+e^{j} \mid t^{j}\right) .
$$

Notice that unlike Vohra (1999), there is no mediator in our model, since we are modelling the reality in which the members of a coalition seldom consult with an outsider (mediator) in carrying out their own agreement.

Let $\hat{F}^{S}$ be the set of all feasible, private measurable and Bayesian incentive-compatible strategy bundles:

$$
\hat{F}^{S}:=\left\{z^{S} \in F^{\prime S} \mid z^{S} \text { satisfies(4) }\right\} .
$$

We remark that $\mathbf{0} \in \hat{F}^{S}$, in particular each set $\hat{F}^{S}$ is nonempty.

The consumers play a cooperative game during the interim period. A descriptive solution of the game is a feasible and coalitionally stable strategy bundle $z^{*}$. Here, the feasibility means that $z^{*} \in \hat{F}^{N}$. By making precise the coalitional stability condition in two different ways, we obtain two versions of the interim core concept. The first (Definition 2.2) is a variant of Wilson's (1978) coarse core concept, modified so that it becomes appropriate in the private information case.

Definition 2.2. A Bayesian incentive-compatible coarse core net-trade plan of a Bayesian pure exchange economy $\mathcal{E}_{p e}$ is a strategy bundle $z^{*}$, such that

(i) $z^{*} \in \hat{F}^{N}$; and

(ii) it is not true that

$$
\begin{aligned}
& \exists S \in \mathcal{N}: \exists z^{S} \in \hat{F}^{S}: \exists E \in \bigwedge_{j \in S} \mathcal{T}^{j}: E \neq \emptyset: \forall t \in E: \forall j \in S: \\
& E u^{j}\left(z^{j}+e^{j} \mid t^{j}\right)>E u^{j}\left(z^{* j}+e^{j} \mid t^{j}\right) .
\end{aligned}
$$


The members of $\wedge_{j \in S} \mathcal{T}^{j}$ are the events that all consumers in $S$ can discern without exchange of their private information. Defecting coalition $S$ forms at a commonly discernable event $E$, if there exists a private measurable and Bayesian incentive-compatible strategy bundle $z^{S}$ with which every member in $S$ improves upon $z^{*}$ at every state in $E$. Notice that in the present type-profile approach,

$$
\bigwedge_{j \in S} \mathcal{T}^{j}= \begin{cases}\mathcal{T}^{i} & \text { if } S=\{i\}, \\ \{\emptyset, T\} & \text { if } \# \leq 2 .\end{cases}
$$

The notion of interim-utility improvement in Definition 2.2 (ii) might be too strong, making the core concept too weak, since it requires improvement even at states which some agents, according to their private information, know do not prevail. However, this definition has the merit that it enables us to avoid all complicated issues related to the information-revelation caused merely by agents' decision to join the coalition. Member $j$ would reveal the information that his type is not $t^{j}$ merely by deciding to join the coalition and agreeing to the defecting strategy $z^{S}$, if his conditional expected utility of $z^{j}+e^{j}$ given $t^{j}$ were lower than his conditional expected utility of $z^{* j}+e^{j}$ given $t^{j}$. In the context of Definition 2.2 (ii), private information cannot be revealed in this way, because in the mutually discernible event $E$, each individual knows that everyone improves upon the contract $z^{*}$ at all states. We will come back to this point in Section 6 where we discuss information-revelation through coalition formation in a specific case.

The second concept (Definition 2.3) may be considered a version of Wilson's (1978) fine core concept, but the two are substantially different: While Wilson's fine core allows a blocking coalition to use an admissible communication system (in short, the members of a coalition can opt to partially pool their private information), we are interested in the private information case in the absence of a mechanism for credible pooling or of a mediator. In our case, therefore, a core plan $z^{*}$ remains private measurable. We retain the strong coalitional stability condition of the fine core that no coalition at any possible type profile can improve upon $z^{*}$.

Definition 2.3. A Bayesian incentive-compatible interim core net-trade plan of a Bayesian pure exchange economy $\mathcal{E}_{p e}$ is a strategy bundle $z^{*}$, such that

(i) $z^{*} \in \hat{F}^{N}$; and

(ii) it is not true that

$$
\exists S \in \mathcal{N}: \exists z^{S} \in \hat{F}^{S}: \exists t^{S} \in T^{S}: \forall j \in S: E u^{j}\left(z^{j}+e^{j} \mid t^{j}\right)>E u^{j}\left(z^{* j}+e^{j} \mid t^{j}\right) .
$$

There are two aspects of the present definition of the fine core that distinguish it from Wilson's fine core. The first aspect is that although this definition may be considered a version of Wilson's fine core, it does not assume communication among agents for pooling information. This is why the private measurability is imposed. The nature of fineness comes from the requirement of a strong coalitional stability as opposed to a weaker stability requirement of the coarse core. The second aspect is that instead of restricting objections to a "commonly discernible" event among the members of the coalition, we impose incentive compatibility. 


\section{Results}

We first present a positive existence result on the Bayesian incentive-compatible coarse core net-trade plan. A function $f: \mathbf{R}_{+}^{l} \rightarrow \mathbf{R}$ is called weakly monotone, if

$$
\left[c, c^{\prime} \in \mathbf{R}_{+}^{l}, c \leq c^{\prime}\right] \Rightarrow f(c) \leq f\left(c^{\prime}\right) .
$$

Proposition 3.1. Let $\mathcal{E}_{\text {pe }}$ be a Bayesian pure exchange economy. Assume for each consumer $j$ that his von Neumann-Morgenstern utility function $u^{j}(\cdot, t)$ is continuous, concave, and weakly monotone in $\mathbf{R}_{+}^{l}$ for every $t \in T$. Then there exists a Bayesian incentive-compatible coarse core net-trade plan.

Remark 3.2. In our proof of Proposition 3.1 (Section 5), we show the existence of an interim Bayesian incentive-compatible coarse core net-trade plan $z^{*}$ which is exactly attained in the grand coalition, that is,

$$
\sum_{j \in N} z^{* j}(t)=\mathbf{0}, \quad \text { for every } t \in T,
$$

while allowing for the broad range of strategies (the weak inequality (2)) for all possible blocking coalitions.

Remark 3.3. Vohra's example of an empty Bayesian incentive-compatible coarse core (Vohra, 1999, Example 3.2, pp. 136-138) is crucially based on his postulate that for a plan $z^{S}$ of coalition $S$, each strategy $z^{j}$ is a function of $t^{S} \in T^{S}$, rather than of $t^{j} \in T^{j}$. Vohra's setup requires the presence of a mediator who, by collecting private information, enlarges the set of possible blocking strategies, thereby making blocking coalition-formation easier.

We turn to the Bayesian incentive-compatible interim core. It is easy to establish that when $l=1$, the zero net-trade plan $t \mapsto \mathbf{0}$ (resulting in the initial endowment function $t \mapsto$ $\left\{e^{j}\left(t^{j}\right)\right\}_{j \in N}$ as the final allocation plan) is the unique Bayesian incentive-compatible interim core plan of a Bayesian pure exchange economy. Indeed, by the individual rationality, a core plan has to yield at least an interim utility allocation $\left\{E u^{j}\left(e^{j} \mid t^{j}\right)\right\}_{j \in N}$ for each type profile $t$. But no coalition $S$ at any type profile $t^{S}$ can improve upon this, since if a member can improve at $t^{S}$ then at least one other member will be worse off at $t^{S}$ in the light of the fact that there is no give and take in the case $l=1$.

For $l \geq 2$, the Bayesian incentive-compatible interim core may be empty, even for a Bayesian pure exchange economy satisfying all the neoclassical convexity assumptions. See, e.g., Hahn and Yannelis (1997, Corollary 8.2.3) for an example of an economy with an empty Bayesian incentive-compatible interim core. The negative result is true even for some economies with linear von Neumann-Morgenstern utility functions. This negative result makes a contrast to nonemptiness of the Bayesian incentive-compatible ex ante core (see, e.g., Ichiishi and Idzik (1996) for the role of linearity of the utility functions in dealing with Bayesian incentive compatibility). Vohra (1999, Example 2.1, pp. 131-132) showed this negative result in his example of a market for a single indivisible lemon. The following example is a minor variant of Vohra's example, obtained by removing the indivisibility. 
Example 3.4. Consider the following two-consumer, two-commodity Bayesian pure exchange economy.

$$
N=\{1,2\}, \quad T^{1}=\{l, h\}, \quad T^{2}=\left\{t^{2}\right\} .
$$

The type-profile space $T:=T^{1} \times T^{2}$ is identified with $T^{1}$. Consumer 1 is the seller of divisible commodity 1 , and consumer 2 is the buyer. The seller knows the quality of commodity 1 , but the buyer does not. The second commodity is money. The consumption set for each consumer is $\mathbf{R}_{+}^{2}$. The initial endowment function on $T$ is a constant function,

$$
\forall t \in T: e^{1}(t)=\left(\begin{array}{l}
1 \\
0
\end{array}\right), \quad e^{2}(t)=\left(\begin{array}{l}
0 \\
w
\end{array}\right),
$$

where $w \geq 6$. We formulate the quality of commodity 1 in terms of the state-dependent von Neumann-Morgenstern utility function. In state $l$, commodity 1 is of low quality, giving rise to no utility. In state $h$, commodity 1 is of high quality, giving rise to positive utility. The utility functions are given by:

$$
\begin{aligned}
& u^{1}(c, t):= \begin{cases}c_{2} & \text { if } t=l \\
10 c_{1}+c_{2} & \text { if } t=h,\end{cases} \\
& u^{2}(c, t):= \begin{cases}c_{2} & \text { if } t=l \\
15 c_{1}+c_{2} & \text { if } t=h .\end{cases}
\end{aligned}
$$

The ex ante probability held by the uninformed buyer is the uniform probability,

$$
\pi^{2}\left(l, t^{2}\right)=\pi^{2}\left(h, t^{2}\right)=\frac{1}{2} .
$$

Then, this economy has no Bayesian incentive-compatible interim core net-trade plan. Section 5 will provide a proof of this nonexistence result.

Remark 3.5. The key idea in this couterexample may succinctly be captured by the following observation. The no-trade interim utility allocation is

$$
\begin{aligned}
& u^{1}\left(e^{1}, t\right)= \begin{cases}0 & \text { if } t=l \\
10 & \text { if } t=h\end{cases} \\
& E u^{2}\left(e^{2}\right)=w .
\end{aligned}
$$

The constant strategy bundle $z \in \hat{F}^{N}$ given by

$$
\begin{aligned}
& z^{1}(l)=z^{1}(h):=\left(\begin{array}{c}
-1 \\
6
\end{array}\right), \\
& z^{2}\left(t^{2}\right):=-z^{1}(l)
\end{aligned}
$$


gives rise to the interim utility allocation,

$$
\begin{aligned}
& u^{1}\left(z^{1}+e^{1}, t\right)= \begin{cases}6 & \text { if } t=l \\
6 & \text { if } t=h\end{cases} \\
& E u^{2}\left(z^{2}+e^{2}\right)=1.5+w .
\end{aligned}
$$

The no-trade utility allocation is blocked by $N$ at $t=l$ via $z$, and the utility allocation of $z$ is blocked by $\{1\}$ at $t=h$ via the no-trade.

Remark 3.5 suggests the fact that a Bayesian incentive-compatible interim core strategy bundle may not exist, because a utility allocation is blocked by a coalition at a particular type profile $\left(S, t^{S}\right)$, and another utility allocation which is stable against $\left(S, t^{S}\right)$ is blocked by a coalition at another type profile $\left(S^{\prime}, t^{\prime} S^{\prime}\right)$. In order to guarantee the existence of a core strategy bundle, therefore, we need to explore the effects of different type profiles. The rest of this section will explore conditions among effects of different type profiles under which a core strategy bundle does exist.

For a clear-cut result, we will postulate that von Neumann-Morgenstern utility functions are affine linear (Assumption 3.6). In the following, commodity bundles $c^{j}+e^{j}\left(t^{j}\right)$ are understood as $l$-dimensional column vectors.

Assumption 3.6 (risk neutrality). For each consumer $j$ and each type $t^{j}$, there exist a nonnegative row vector $a^{j}\left(t^{j}\right)$ and a scaler $b^{j}\left(t^{j}\right)$, such that

$$
E u^{j}\left(c^{j}+e^{j} \mid t^{j}\right)=a^{j}\left(t^{j}\right)\left(c^{j}+e^{j}\left(t^{j}\right)\right)+b^{j}\left(t^{j}\right), \quad \text { for all } c^{j} .
$$

Here, $a^{j}\left(t^{j}\right)$ is the vector of marginal (interim) utilities given type $t^{j}$, postulated to be nonnegative. For each coalition $S$, define the coalitionally feasible choice space by

$$
\begin{aligned}
C_{0}^{S}: & =\left\{\begin{array}{l|l}
c^{S} \in \mathbf{R}^{l \cdot \# S} & \begin{array}{l}
\forall j \in S: \forall t^{j} \in T^{j}: c^{j}+e^{j}\left(t^{j}\right) \geq \mathbf{0} \\
\sum_{j \in S} c^{j} \leq \mathbf{0}
\end{array}
\end{array}\right\} \\
& =\left\{\begin{array}{l|l}
c^{S} \in \mathbf{R}^{l \cdot \# S} & \begin{array}{l}
\forall j \in S: c^{j}+\underline{e}^{j} \geq \mathbf{0} \\
\sum_{j \in S} c^{j} \leq \mathbf{0}
\end{array}
\end{array}\right\},
\end{aligned}
$$

where $\underline{e}_{h}^{j}:=\min \left\{e_{h}^{j}\left(t^{j}\right) \mid t^{j} \in T^{j}\right\}$, in short, $\underline{e}^{j}:=\inf _{t^{j} \in T^{j}} e^{j}\left(t^{j}\right)$. The set $C_{0}^{S}$ is nonempty; indeed, $\mathbf{0} \in C_{0}^{S}$.

An agent of economy $\mathcal{E}_{p e}$ is defined as a consumer together with his type, $\left(j, t^{j}\right)$; denote by $A$ the set of all agents,

$$
A:=\left\{\left(j, t^{j}\right) \mid j \in N, t^{j} \in T^{j}\right\} .
$$

An admissible blocking coalition is a coalition of agents in which at most one agent represents each consumer; denote by $\mathcal{B}_{0}$ the family of all admissible blocking coalitions,

$$
\mathcal{B}_{0}:=\left\{B \subset A \mid\left[\left(i, t^{i}\right),\left(j, t^{j}\right) \in B, t^{i} \neq t^{j}\right] \Rightarrow i \neq j\right\} .
$$


Thus, consumer-coalition $S$ forms as a blocking coalition in $\mathcal{E}_{p e}$ at type profile $\bar{t}^{S}$, iff the admissible agent-coalition $B:=\left\{\left(j, \bar{t}^{j}\right) \in A \mid j \in S\right\}$ forms. For $B \in \mathcal{B}_{0}$, let $S(B)$ be the set of those consumers represented by the agents $B$,

$$
S(B):=\left\{j \in N \mid \exists t^{j} \in T^{j}:\left(j, t^{j}\right) \in B\right\} .
$$

Also, let $t^{j}(B)$ be the consumer $j$ 's type for which $\left(j, t^{j}(B)\right) \in B$.

In the light of the linearity assumption (Assumption 3.6), we may define the maximal coalitional gain for each $B \in \mathcal{B}_{0}$,

$$
v(B):=\max \left\{\begin{array}{l|l}
\sum_{j \in S(B)} a^{j}\left(t^{j}(B)\right) c^{j} & \begin{array}{l}
c^{S} \in C_{0}^{S(B)}, \\
\forall j \in S(B): a^{j}\left(t^{j}(B)\right) c^{j} \geq 0
\end{array}
\end{array}\right\} .
$$

It is achieved with net trades within $S(B)$ that are individually feasible $\left(c^{j}+\underline{e}^{j} \geq \mathbf{0}\right)$, coalitionally attainable $\left(\sum_{j \in S(B)} c^{j} \leq \mathbf{0}\right)$, and individually rational $\left(a^{j}\left(t^{j}(B)\right) c^{j} \geq 0\right)$. The concept of maximal coalitional gain $v(B)$ assumes transfer of utilities among the players, but these numerical values are needed only in a quantitative condition (Assumption 3.7) of the existence theorem (Theorem 3.8). Notice that the gain $v(B)$ depends upon $\left\{\underline{e}^{j}\right\}_{j \in S(B)}$. We will discuss Assumption 3.7 after presentation of Theorem 3.8.

Assumption 3.7 (Possibility of utility enhancing multilateral trades). For all $\left\{\lambda_{B}\right\}_{B \in \mathcal{B}_{0}}$ $\left(\subset \mathbf{R}_{+}\right)$and all $\left\{\mu^{j}\right\}_{j \in N}\left(\subset \mathbf{R}_{+}^{l}\right)$ for which

$$
\forall i, j \in N: \sum_{B \in \mathcal{B}_{0}: S(B) \ni i} \lambda_{B} a^{i}\left(t^{i}(B)\right)+\mu^{i}=\sum_{B \in \mathcal{B}_{0}: S(B) \ni j} \lambda_{B} a^{j}\left(t^{j}(B)\right)+\mu^{j},
$$

it follows that

$$
\sum_{B \in \mathcal{B}_{0}} \lambda_{B} v(B) \leq \sum_{j \in N} \mu^{j} \underline{\underline{e}}^{j}
$$

Theorem 3.8. Let $\mathcal{E}_{\text {pe }}$ be a Bayesian pure exchange economy which satisfies Assumptions 3.6 and 3.7. Then a Bayesian incentive-compatible interim core net-trade plan of $\mathcal{E}_{\text {pe }}$ exists.

To clarify the meaning of Assumption 3.7, consider for example the economy $\mathcal{E}_{p e}$ with two consumers $(N=\{1,2\})$. If $v(B)=0$ for all $B \in \mathcal{B}_{0}$, then the assumption is automatically satisfied, so the Bayesian incentive-compatible interim core is nonempty. Otherwise, for each consumer $j$, let $K^{j}$ be the cone spanned by consumer $j$ 's marginal (interim) utility vectors,

$$
K^{j}:=\left\{\sum_{B \in \mathcal{B}_{0}: S(B) \ni j} \lambda_{B} a^{j}\left(t^{j}(B)\right) \in \mathbf{R}_{+}^{l} \mid\left(\forall B \in \mathcal{B}_{0}: S(B) \ni j\right): \lambda_{B} \geq 0\right\} .
$$

If there exists nonzero $\left\{\lambda_{B}\right\}_{B \in \mathcal{B}_{0}}$ which gives rise to a member in $K^{1} \cap K^{2}$, then together with $\mu^{j}=\mathbf{0}$ for all $j \in N$, it satisfies constraint (5). So the required inequal- 
ity (6) is not satisfied unless $v(B)=0$ for all $B \in \mathcal{B}_{0}$, and Theorem 3.8 cannot be applied.

If $K^{1} \cap K^{2}=\{\mathbf{0}\}$, then for any nontrivial $\lambda_{B}$ 's and $\mu^{j}$ 's to satisfy (5), some $\mu^{j}$ must be nonzero, and if the corresponding $\underline{e}^{j}$ is large, the required inequality (6) is satisfied, and the Bayesian incentive-compatible interim core is nonempty.

Here is one economic interpretation of Assumption 3.7. Define

$$
v:=\sum_{B \in \mathcal{B}_{0}: S(B) \ni j} \lambda_{B} a^{j}\left(t^{j}(B)\right)+\mu^{j} \in \mathbf{R}_{+}^{l} .
$$

The vector $v$ is independent of $j$ in view of (5). Suppose that the society values each agent-coalition $B$ as $\lambda_{B}$, and each commodity $h$ as $v_{h}$. Then, by holding a unit of the $h$ th initial endowment, consumer $j$ enjoys two attributes: One is the increase in utility by possessing it as a member of various coalitions, $\sum_{B \in \mathcal{B}_{0}: S(B) \ni j} \lambda_{B} a_{h}^{j}\left(t^{j}(B)\right)$, and the other is its excess value as an asset, $\mu_{h}^{j}:=v_{h}-\sum_{B \in \mathcal{B}_{0}: S(B) \ni j} \lambda_{B} a_{h}^{j}\left(t^{j}(B)\right)$. Assumption 3.7 says that in this situation, the society's maximal utility gain, re-scaled in order to take into account the value of each coalition, $\sum_{B \in \mathcal{B}_{0}} \lambda_{B} v(B)$, is achieved by the total excess value of the initial endowments, $\sum_{j \in N} \mu^{j} \underline{e}^{j}$.

\section{Examples}

In this section, we will re-investigate the example of the single lemon market (Example 3.4) in the light of the existence Theorem 3.8, and then study several further examples that are obtained by modifying the single lemon market example.

Example 4.1. The same example of the single divisible lemon market as in Example 3.4. In this case,

$$
\begin{aligned}
& a^{1}(l)=(0,1), \\
& a^{1}(h)=(10,1), \\
& a^{2}\left(t^{2}\right)=(7.5,1),
\end{aligned}
$$

so $K^{2} \subset K^{1}$, and Assumption 3.7 is violated. See Fig. 1. This partially explains nonexistence of the Bayesian incentive-compatible interim core net-trade plan in this example.

To facilitate the discussions in the following Examples 4.2-4.6, we will see more closely how Assumption 3.7 is violated here. For simplicity, set $(N, l):=\left\{(1, l),\left(2, t^{2}\right)\right\} \in \mathcal{B}_{0}$, and $(N, h):=\left\{(1, h),\left(2, t^{2}\right)\right\} \in \mathcal{B}_{0}$. In general, $v(B)=0$ for all $B \in \mathcal{B}_{0}$ for which $\# S(B)=1$. So, in considering $\lambda_{B}$ 's and $\mu^{j}$ 's satisfying condition (5), we may assume $\lambda_{B}=0$ for all $B$ for which $\# S(B)=1$. By homogeneity, we may also set $\lambda:=\lambda_{(N, l)}$, and assume that $\lambda_{(N, h)}=1-\lambda$. Then condition (5) becomes

$$
\begin{aligned}
& 10(1-\lambda)+\mu_{1}^{1}=7.5+\mu_{1}^{2} \\
& 1+\mu_{2}^{1}=1+\mu_{2}^{2} .
\end{aligned}
$$




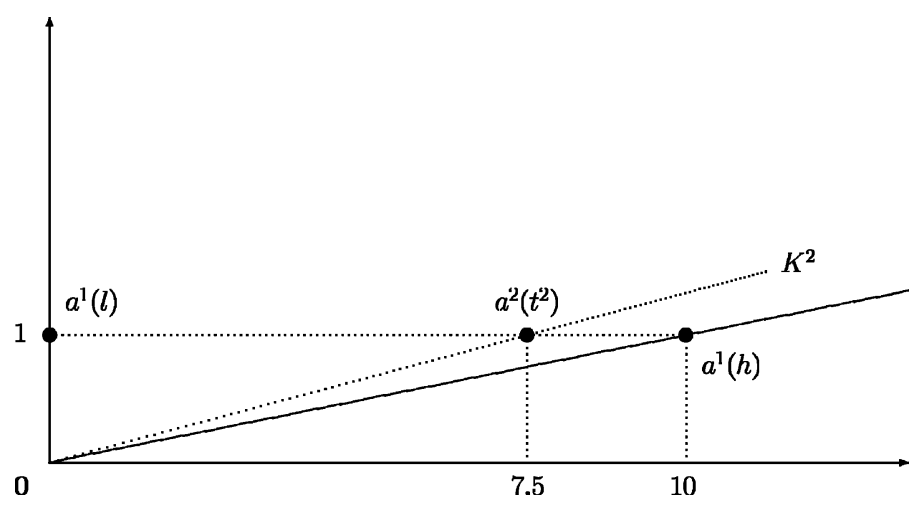

Fig. 1. Examples 3.4 and 4.1. Market for lemons.

On the other hand,

$$
v(B)= \begin{cases}7.5 & \text { if } B=(N, l) \\ 0 & \text { if } B=(N, h)\end{cases}
$$

(the maximal gain $v((N, l))$ is achieved by the net trades, $c^{1}=(-1,0), c^{2}=-c^{1}$, which are individually feasible, coalitionally attainable and individually rational, and $v((N, h))$ is achieved by the no-trade), so the required inequality (6) becomes

$$
7.5 \lambda \leq \mu_{1}^{1}+\mu_{2}^{2} w .
$$

Thus the Assumption 3.7 is violated for

$$
\lambda=0.25, \mu^{1}=\mu^{2}=\mathbf{0} .
$$

Example 4.2. We look at variants of the one-informed-consumer, one-uninformed-consumer example (Example 3.4). There are two commodities $(l=2)$. We specify $N, T$, and $u^{1}$ as in Example 3.4, but consider different data on $E u^{2}: \mathbf{R}_{+}^{2} \rightarrow \mathbf{R}$ (still assumed to be affine linear, so that $\left.E u^{2}(c)=a^{2}\left(t^{2}\right) c\right)$ and on $e^{j}:=\left(e_{1}^{j}, e_{2}^{j}\right) \in \mathbf{R}_{+}^{2}, j \in N$.

As in Example 4.1 we define for simplicity, $(N, l):=\left\{(1, l),\left(2, t^{2}\right)\right\}$, and $(N, h):=$ $\left\{(1, h),\left(2, t^{2}\right)\right\}$. Also as in Example 4.1, in considering $\lambda_{B}$ 's and $\mu^{j}$ 's satisfying condition (5), we may assume $\lambda_{B}=0$ for all $B$ for which $\# S(B)=1$, and may set $\lambda:=\lambda_{(N, l)}$, and $\lambda_{(N, h)}=1-\lambda$.

Case 1. Suppose $a^{2}\left(t^{2}\right)=(\alpha, 1)$, for some $\alpha>10$. See Fig. 2. Then, $K^{1} \cap K^{2}=\{\boldsymbol{0}\}$. If $10 e_{1}^{1} \leq e_{2}^{2}$, then

$$
v(B)= \begin{cases}\alpha e_{1}^{1} & \text { for } B=(N, l), \\ (\alpha-10) e_{1}^{1} & \text { for } B=(N, h)\end{cases}
$$




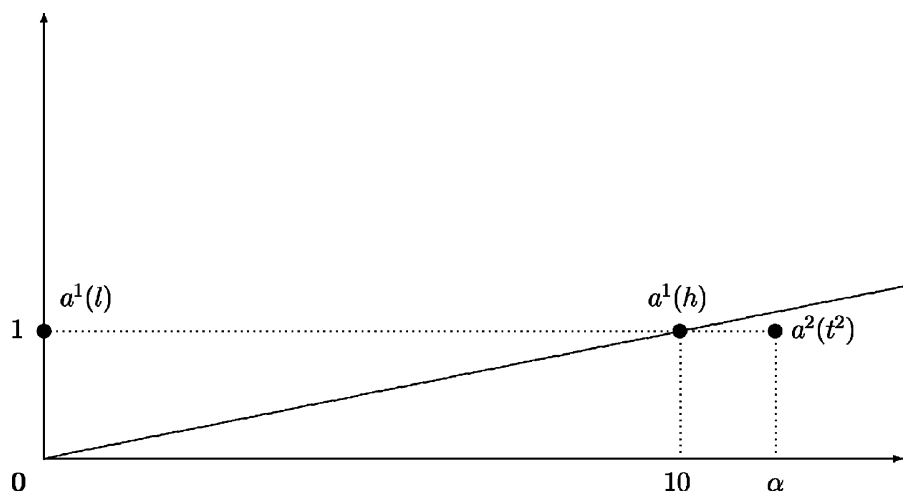

Fig. 2. Example 4.2, Case 1. $a^{2}\left(t^{2}\right)=(\alpha, 1), \alpha>10$.

(both maximal gains $v(B), B=(N, l),(N, h)$, can be achieved by the individually feasible, coalitionally attainable and individually rational net trades $\left.c^{1}=\left(-e_{1}^{1}, 10 e_{1}^{1}\right), c^{2}=-c^{1}\right)$. Coefficients $\lambda$ and $\mu^{j}$, s satisfy (5), iff

$$
\begin{aligned}
& (1-\lambda) 10+\mu_{1}^{1}=\alpha+\mu_{1}^{2} \\
& 1+\mu_{2}^{1}=1+\mu_{2}^{2} .
\end{aligned}
$$

The required inequality (6) becomes

$$
\lambda \alpha e_{1}^{1}+(1-\lambda)(\alpha-10) e_{1}^{1} \leq \mu^{1} e^{1}+\mu^{2} e^{2},
$$

that is,

$$
\left[\alpha-(1-\lambda) 10-\mu_{1}^{1}\right] e_{1}^{1} \leq \mu_{2}^{1} e_{2}^{1}+\mu^{2} e^{2} .
$$

But by (5), the left-hand side is equal to $-\mu_{1}^{2} e_{1}^{1}$, so the required inequality is always satisfied. If $10 e_{1}^{1}>e_{2}^{2}$, then

$$
v(B)= \begin{cases}\alpha e_{1}^{1} & \text { for } B=(N, l), \\ (\alpha-10) \frac{1}{10} e_{2}^{2}<(\alpha-10) e_{1}^{1} & \text { for } B=(N, h),\end{cases}
$$

so given (5), the required condition is a fortiori satisfied.

Thus, Assumption 3.7 is satisfied for arbitrary initial endowment vectors, $e^{1}, e^{2}$.

Case 2. Suppose $a^{2}\left(t^{2}\right)=(15,0)$. (One can easily draw figures analogous to Fig. 1 for the present Case 2 and for the subsequent examples.) Then, $K^{1} \cap K^{2}=\{\mathbf{0}\}$. If $10 e_{1}^{1} \leq e_{2}^{2}$,

$$
v(B)= \begin{cases}15 e_{1}^{1}+e_{2}^{2}, & \text { for } B=(N, l), \\ 5 e_{1}^{1}+e_{2}^{2}, & \text { for } B=(N, h)\end{cases}
$$

(both maximal gains can be achieved by the individually feasible, coalitionally attainable and individually rational net trades $\left.c^{1}=\left(-e_{1}^{1}, e_{2}^{2}\right), c^{2}=-c^{1}\right)$. Coefficients $\lambda$ and $\mu^{j}$ 's 
satisfy (5), iff

$$
\begin{aligned}
& (1-\lambda) 10+\mu_{1}^{1}=15+\mu_{1}^{2} \\
& 1+\mu_{2}^{1}=\mu_{2}^{2} .
\end{aligned}
$$

The required inequality (6) becomes

$$
\lambda\left(15 e_{1}^{1}+e_{2}^{2}\right)+(1-\lambda)\left(5 e_{1}^{1}+e_{2}^{2}\right) \leq \mu^{1} e^{1}+\mu^{2} e^{2},
$$

that is,

$$
\left[15-(1-\lambda) 10-\mu_{1}^{1}\right] e_{1}^{1}+e_{2}^{2} \leq \mu_{2}^{1} e_{2}^{1}+\mu^{2} e^{2} .
$$

In view of (5), this is

$$
-\mu_{1}^{2} e_{1}^{1}+e_{2}^{2} \leq \mu_{2}^{1} e_{2}^{1}+\mu_{1}^{2} e_{1}^{2}+\left(1+\mu_{2}^{1}\right) e_{2}^{2},
$$

which is always satisfied.

$$
\begin{aligned}
& \text { If } 10 e_{1}^{1}>e_{2}^{2}, \\
& v(B)= \begin{cases}15 e_{1}^{1}+e_{2}^{2}, & \text { for } B=(N, l), \\
5 \cdot \frac{1}{10} e_{2}^{2}+e_{2}^{2}<5 e_{1}^{1}+e_{2}^{2}, & \text { for } B=(N, h),\end{cases}
\end{aligned}
$$

so given (5), the required condition is a fortiori satisfied.

Thus, Assumption 3.7 is satisfied for arbitrary initial endowment vectors, $e^{1}, e^{2}$.

Case 3. Suppose $a^{2}\left(t^{2}\right)=(7.5,0)$. Then, $K^{1} \cap K^{2}=\{\boldsymbol{0}\}$, and

$$
v(B)= \begin{cases}7.5 e_{1}^{1}+e_{2}^{2}, & \text { if } B=(N, l), \\ e_{2}^{2}, & \text { if } B=(N, h)\end{cases}
$$

(the second identity is due to the individual rationality of consumer 2). Coefficients $\lambda$ and $\mu^{j}$ 's satisfy (5), iff

$$
\begin{aligned}
& (1-\lambda) 10+\mu_{1}^{1}=7.5+\mu_{1}^{2} \\
& 1+\mu_{2}^{1}=\mu_{2}^{2} .
\end{aligned}
$$

The required inequality (6) becomes

$$
\lambda\left(7.5 e_{1}^{1}+e_{2}^{2}\right)+(1-\lambda) e_{2}^{2} \leq \mu^{1} e^{1}+\mu^{2} e^{2} .
$$

On the one hand, condition (6) is satisfied, if $e_{1}^{1}=0$. On the other hand, if $e_{1}^{1}>0$, condition (6) is violated by $\lambda=0.25, \mu^{1}=\mathbf{0}$ and $\mu^{2}=(0,1)$. Thus, Assumption 3.7 is satisfied, iff $e_{1}^{1}=0$, that is, iff consumer 1 does not initially possess commodity 1 .

Example 4.3. This is another variant of the one-informed consumer, one-uniformed consumer example (Example 3.4). We introduce the third commodity which is also a lemon, ${ }^{3}$

\footnotetext{
${ }^{3}$ In general, if there are more than two possible lemons, the type space needs to be expanded to take into account the quality of the second lemon/peach. Actually, due to the perfect (negative) correlation, we do not have to expand the dimension in the present formulation.
} 
but its quality has the reverse contingency, that is, the quality is high if $t=l$, and is low if $t=h$. Thus, the two lemons (commodities 1 and 3) might kill their destabilizing effects each other. We will see, however, that Assumption 3.7 is still violated. This means that the destabilizing effect of the very asymmetry in information is robust.

Define $a^{j}\left(t^{j}\right) \in \mathbf{R}^{3}$ as in Assumption 3.6. Thus,

$$
\begin{aligned}
& N=\{1,2\}, \quad T^{1}=\{l, h\}, \quad T^{2}=\left\{t^{2}\right\}, \\
& \forall t \in T: e^{1}(t)=\left(\begin{array}{l}
1 \\
0 \\
1
\end{array}\right), \quad e^{2}(t)=\left(\begin{array}{l}
0 \\
w \\
0
\end{array}\right), \\
& a^{1}(l)=(0,1,10), \\
& a^{1}(h)=(10,1,0), \\
& a^{2}\left(t^{2}\right)=(a, 1, b),
\end{aligned}
$$

where $0<a<10$ and $0<b<10$. We will show that this economy does not satisfy Assumption 3.7.

Define $(N, l)$ and $(N, h)$ as in Example 4.1. Also as in Example 4.1, in considering $\lambda_{B}$ 's and $\mu^{j}$,s satisfying condition (5), we may assume $\lambda_{B}=0$ for all $B$ for which $\# S(B)=1$, and may set $\lambda:=\lambda_{(N, l)}$, and $\lambda_{(N, h)}=1-\lambda$. Observe that

$$
v(B)= \begin{cases}a, & \text { if } B=(N, l), \\ b, & \text { if } B=(N, h)\end{cases}
$$

(the maximal gain $v((N, l))$ is achieved by the net trades, $c^{1}=(-1,0,0), c^{2}=-c^{1}$, which are individually feasible, coalitionally attainable and individually rational, and $v((N, h))$ is achieved by the net trades, $\left.c^{1}=(0,0,-1), c^{2}=-c^{1}\right)$.

Condition (5) becomes:

$$
\begin{aligned}
& (1-\lambda) 10+\mu_{1}^{1}=a+\mu_{1}^{2}, \\
& 1+\mu_{2}^{1}=1+\mu_{2}^{2}, \\
& \lambda 10+\mu_{3}^{1}=b+\mu_{3}^{2} .
\end{aligned}
$$

(Compare with the equivalent condition to (5) in Example 4.1; the first of the above three equations corresponds to the fact that commodity 1 is a lemon, and the third equation corresponds to the fact that commodity 3 is a lemon.)

The requirement (condition (6)) becomes:

$$
\lambda a+(1-\lambda) b \leq \mu_{1}^{1}+\mu_{3}^{1}+\mu_{2}^{2} w,
$$

which, in the light of (5), is equivalent to:

$$
10 \leq(1-\lambda) a+\lambda b+\mu_{1}^{2}+\mu_{3}^{2}+\mu_{2}^{2} w .
$$


We will first show that $10<a+b$, if (6) is to be valid for all $\left(\lambda, \mu^{1}, \mu^{2}\right)$ satisfying (5). Indeed, if $10 \geq a+b$, then there exists $\lambda$ satisfying

$$
\begin{aligned}
& (1-\lambda) 10 \geq a \\
& \lambda 10 \geq b .
\end{aligned}
$$

Set $\mu_{1}^{1}=\mu_{3}^{1}=\mu_{2}^{i}=0$, and $\mu_{1}^{2}:=(1-\lambda) 10-a \geq 0, \mu_{3}^{2}:=\lambda 10-b \geq 0$. These $\lambda, \mu^{1}$, $\mu^{2}$ satisfy condition (5). But (6) becomes

$$
\lambda a+(1-\lambda) b \leq 0
$$

which cannot be true.

It suffices to show also that $10>a+b$, if (6) is to be valid for all $\left(\lambda, \mu^{1}, \mu^{2}\right)$ satisfying (5). Indeed, if $10 \leq a+b$, then there exists $\lambda$ satisfying

$$
\begin{aligned}
& (1-\lambda) 10 \leq a \\
& \lambda 10 \leq b,
\end{aligned}
$$

Set $\mu_{1}^{2}=\mu_{3}^{2}=\mu_{2}^{i}=0$, and $\mu_{1}^{1}:=a-(1-\lambda) 10 \geq 0, \mu_{3}^{1}:=b-\lambda 10 \geq 0$. These $\lambda, \mu^{1}$, $\mu^{2}$ satisfy condition (5). But (6) becomes

$$
10 \leq \lambda a+(1-\lambda) b
$$

which cannot be true.

In addition to the negative result on the single lemon market example, we have obtained a partially negative result (Case 3 of Example 4.2) and a totally negative result (Example 4.3). We turn to positive Examples 4.4-4.6 now. We will show in Example 4.4 that a slight modification of the previous examples (Case 3 of Example 4.2, and Example 4.3) guarantees Assumption 3.7. Examples 4.5 and 4.6 are modified Cases 1 and 2 of Example 4.2, and are included here for completeness.

Example 4.4. We turn to Case 3 of Example 4.2 and to Example 4.3, both of which either had no endowment of the first commodity (lemon) by a potential seller (i.e., $e_{1}^{1}=0$ ) or did not satisfy Assumption 3.7 of our theorem. It is worthwhile to see what kind of perturbation is needed to change those examples into ones that satisfy the assumption of the theorem while requiring $e_{1}^{1}>0$. The common element in both of these examples is that the uninformed consumer does not give absolutely higher evaluation of a potential lemon than the informed consumer in high quality state in terms of their conditional expected utilities. Thus, it would be very interesting to check whether our assumption can still be satisfied under these circumstances in a variant of the single lemon model.

In Case 3 of Example 4.2 the second commodity is no longer a "money" but a commodity that gives possibly different degrees of marginal utilities to consumers. It seems immediately clear to us that one of the key factors in this example is that consumer 2 who is a potential "buyer" in the lemon's model must have at least relatively higher conditional marginal utility 
for a potential lemon than consumer 1 who is a potential "seller." We simply modify the example in Case 3 as follows: The initial endowment function on $T$ is a constant function,

$$
\forall t \in T: e^{1}(t)=\left(\begin{array}{l}
e_{1}^{1} \\
e_{2}^{1}
\end{array}\right), \quad e^{2}(t)=\left(\begin{array}{l}
e_{1}^{2} \\
e_{2}^{2}
\end{array}\right) \quad \text { with } e_{1}^{1}>0 \text { and } e_{2}^{2}>0 .
$$

Let $^{4}$

$$
\begin{aligned}
& a^{1}(l)=\left(0, \beta_{l}\right), \\
& a^{1}(h)=\left(\alpha_{h}, \beta_{h}\right), \\
& a^{2}\left(t^{2}\right)=(\alpha, \beta),
\end{aligned}
$$

and

$$
\begin{aligned}
& 0<\alpha<\alpha_{h}, \\
& 0<\beta<\min \left\{\beta_{l}, \beta_{h}\right\} \\
& \operatorname{MRS}_{12}^{2}=\frac{\alpha}{\beta}>\frac{\alpha_{h}}{\beta_{h}}=\operatorname{MRS}_{12}^{1 h} .
\end{aligned}
$$

The remaining specification and notation are as in Example 4.2. Then, $K^{1} \cap K^{2}=\{\mathbf{0}\}$, and we have

$$
\begin{aligned}
& v((N, l))=\beta_{l}\left(\frac{\alpha}{\beta}\right) \min \left\{e_{1}^{1}, \frac{\beta}{\alpha} e_{2}^{2}\right\} \\
& v((N, h))=\beta_{h}\left(\frac{\alpha}{\beta}-\frac{\alpha_{h}}{\beta_{h}}\right) \min \left\{e_{1}^{1}, \frac{\beta}{\alpha} e_{2}^{2}\right\} .
\end{aligned}
$$

Coefficients $\lambda$ and $\mu^{j}$ 's satisfy (5), iff

$$
\begin{aligned}
& (1-\lambda) \alpha_{h}+\mu_{1}^{1}=\alpha+\mu_{1}^{2}, \\
& \lambda \beta_{l}+(1-\lambda) \beta_{h}+\mu_{2}^{1}=\beta+\mu_{2}^{2} .
\end{aligned}
$$

The required inequality (6) becomes

$$
\begin{aligned}
& {\left[\frac{\alpha}{\beta} \beta_{\lambda}-(1-\lambda) \alpha_{h}\right] \min \left\{e_{1}^{1}, \frac{\beta}{\alpha} e_{2}^{2}\right\}} \\
& \quad \leq\left[\alpha-(1-\lambda) \alpha_{h}+\mu_{1}^{2}\right] e_{1}^{1}+\left(\beta_{\lambda}-\beta+\mu_{2}^{1}\right) e_{2}^{1}+\operatorname{Res}(\mu),
\end{aligned}
$$

where

$$
\begin{aligned}
& \beta_{\lambda}:=\lambda \beta_{l}+(1-\lambda) \beta_{h}, \\
& \operatorname{Res}(\mu):=\mu_{1}^{2} e_{1}^{1}+\mu_{2}^{1} e_{2}^{1}+\mu_{1}^{2} e_{1}^{2}+\mu_{2}^{1} e_{2}^{2} .
\end{aligned}
$$

If $(\alpha / \beta) e_{1}^{1}>e_{2}^{2}$, condition (6) becomes:

$$
0 \leq\left(e_{1}^{1}-\frac{\beta}{\alpha} e_{2}^{2}\right)\left[\alpha-(1-\lambda) \alpha_{h}\right]+\operatorname{Res}(\mu),
$$

which need not be satisfied for values of $0 \leq \lambda<1-\left(\alpha / \alpha_{h}\right)$.

\footnotetext{
${ }^{4} \mathrm{MRS}_{12}^{j}$ denotes the consumer $j$ 's marginal rate of substitution of commodity 2 for commodity 1.
} 
On the other hand, if $(\alpha / \beta) e_{1}^{1} \leq e_{2}^{2}$, then condition (6) becomes:

$$
0 \leq\left(\beta_{\lambda}-\beta\right)\left(e_{2}^{2}-\frac{\alpha}{\beta} e_{1}^{1}\right)+\operatorname{Res}(\mu),
$$

which is satisfied for any $0 \leq \lambda \leq 1$ and any $\mu_{i}^{j} \geq 0$.

Therefore, Assumption 3.7 is satisfied for parameter values of $e_{1}^{1}, e_{2}^{2}$, such that $(\alpha / \beta) e_{1}^{1} \leq$ $e_{2}^{2}$. This means that Assumption 3.7 is satisfied when there are sufficiently many units of commodity 2 (in the hand of potential buyer of the potential lemon) so that the buyer has enough to compensate the seller for his transfer of the initial endowment of the lemon to the buyer in state $\left(h, t^{2}\right)$.

At first glance it might seem that the interim core is empty in case of Example 4.4 as the expected conditional marginal utility $\alpha$ of the buyer for a potential lemon is strictly less than the expected conditional marginal utility $\alpha_{h}$ of the seller when it is not a lemon but a peach. Nonetheless, Assumption 3.7 is satisfied and the interim core is nonempty. There are two key elements in Example 4.4.

(a) Despite the lower expected conditional marginal utility of the buyer for a potential lemon, there is another commodity 2 , his expected conditional marginal rate of substitution of which for a potential lemon is kept higher than the expected conditional marginal rate of substitution of the seller so that there is no reversal of the superiority of the expected conditional marginal rate of substitution for a potential lemon depending upon type profiles.

(b) There must be enough initial endowment of the commodity, in the hands of the buyer, that is used to compensate the seller for his transfer of his potential lemon.

Case 3 of Example 4.2 did not satisfy (a). Example 4.3 did satisfy (a) but did not satisfy (b).

Example 4.5. We modify Case 1 of the previous Example 4.2. Intuitively, what is "wrong" about Example 4.1 is that the uninformed consumer does not posses a commodity which the informed consumer badly wishes to have so that there is a very profitable trade between the two. Thus, we introduce a third commodity which "enhances" the utility of the informed consumer without affecting that of the uninformed consumer. The consumption set for each consumer is $\mathbf{R}_{+}^{3}$. The initial endowment function on $T$ is a constant function,

$$
\forall t \in T: e^{1}(t)=\left(\begin{array}{c}
1 \\
0 \\
e_{3}^{1}
\end{array}\right), \quad e^{2}(t)=\left(\begin{array}{c}
0 \\
w \\
e_{3}^{2}
\end{array}\right)
$$

Let

$$
\begin{aligned}
& a^{1}(l)=\left(0,1, \beta_{l}\right), \\
& a^{1}(h)=\left(10,1, \beta_{h}\right), \\
& a^{2}\left(t^{2}\right)=(\alpha, 1,0),
\end{aligned}
$$


and assume

$$
\beta_{h} e_{3}^{2} \geq 10 \text {. }
$$

The remaining specification and notation are as in Case 1 . Then, $K^{1} \cap K^{2}=\{\mathbf{0}\}$, and

$$
\begin{aligned}
& v((N, l))=\beta_{l} e_{3}^{2}+\alpha, \\
& v((N, h))=-10+\beta_{h} e_{3}^{2}+\alpha .
\end{aligned}
$$

Coefficients $\lambda$ and $\mu^{j}$, s satisfy (5), iff

$$
\lambda\left(0,1, \beta_{l}\right)+(1-\lambda)\left(10,1, \beta_{h}\right)+\mu^{1}=(\alpha, 1,0)+\mu^{2} .
$$

The required inequality (6) becomes

$$
0 \leq \mu_{3}^{1}\left(e_{3}^{1}+e_{3}^{2}\right)+\mu_{2}^{2} w .
$$

Thus, Assumption 3.7 is always satisfied. Note that $e_{1}^{1}=1$ so that the informed consumer has a single potential lemon as in Examples 3.4 and 4.1, and still Assumption 3.7 is satisfied. The specific circumstance in this example that contributes to fulfillment of Assumption 3.7 is that there is a third commodity which highly enhances the utility of the informed consumer if it is provided to him in exchange for the single potential lemon whose consumption in turn by the uninformed consumer highly enhances his utility.

Example 4.6. We turn to Case 2 of Example 4.2. Noting that in Example 4.5 the second commodity "money" does not play any role in guaranteeing the fulfillment of the condition, a crucial aspect is to have a commodity like the third one in the example. Thus, we modify Case 2 so as to have a more general setting than the previous one with the second commodity eliminated. Thus, the consumption set for each consumer is $\mathbf{R}_{+}^{2}$. The initial endowment function on $T$ is a constant function,

$$
\forall t \in T: e^{1}(t)=\left(\begin{array}{l}
e_{1}^{1} \\
e_{2}^{1}
\end{array}\right), \quad e^{2}(t)=\left(\begin{array}{c}
e_{1}^{2} \\
e_{2}^{2}
\end{array}\right), \quad \text { with } e_{1}^{1}>0, e_{2}^{2}>0 .
$$

Moreover, we assume

$$
0<\frac{\alpha}{\beta} e_{1}^{1} \leq e_{2}^{2}
$$

Let

$$
\begin{aligned}
& a^{1}(l)=\left(\alpha_{l}, \beta_{l}\right), \\
& a^{1}(h)=\left(\alpha_{h}, \beta_{h}\right), \\
& a^{2}\left(t^{2}\right)=(\alpha, \beta),
\end{aligned}
$$

and

$$
\begin{aligned}
& 0<\max \left\{\alpha_{l}, \alpha_{h}\right\}<\alpha, \\
& 0<\beta<\min \left\{\beta_{l}, \beta_{h}\right\}, \\
& \operatorname{MRS}_{12}^{1 l}=\frac{\alpha_{l}}{\beta_{l}}<\operatorname{MRS}_{12}^{1 h}=\frac{\alpha_{h}}{\beta_{h}}<\operatorname{MRS}_{12}^{2}=\frac{\alpha}{\beta} .
\end{aligned}
$$


The remaining specification and notation are as in Example 4.2. Then, $K^{1} \cap K^{2}=\{\mathbf{0}\}$, and we have

$$
\begin{aligned}
& v((N, l))=\beta_{l}\left(\frac{\alpha}{\beta}-\frac{\alpha_{l}}{\beta_{l}}\right) \min \left\{e_{1}^{1}, \frac{\beta}{\alpha} e_{2}^{2}\right\} \\
& v((N, h))=\beta_{h}\left(\frac{\alpha}{\beta}-\frac{\alpha_{h}}{\beta_{h}}\right) \min \left\{e_{1}^{1}, \frac{\beta}{\alpha} e_{2}^{2}\right\} .
\end{aligned}
$$

Coefficients $\lambda$ and $\mu^{j}$, s satisfy (5), iff

$$
\lambda\left(\alpha_{l}, \beta_{l}\right)+(1-\lambda)\left(\alpha_{h}, \beta_{h}\right)+\mu^{1}=(\alpha, \beta)+\mu^{2} .
$$

The required inequality (6) becomes

$$
0 \leq\left(\beta_{\lambda}-\beta\right)\left(e_{2}^{2}-\frac{\alpha}{\beta} e_{1}^{1}\right)+\operatorname{Res}(\mu),
$$

where

$$
\begin{aligned}
& \beta_{\lambda}:=\lambda \beta_{l}+(1-\lambda) \beta_{h}, \\
& \operatorname{Res}(\mu):=\mu_{1}^{2} e_{1}^{1}+\mu_{2}^{1} e_{2}^{1}+\mu_{1}^{2} e_{1}^{2}+\mu_{2}^{1} e_{2}^{2} .
\end{aligned}
$$

The inequality is always satisfied. Therefore, Assumption 3.7 is satisfied for parameter values of $e_{1}^{1}, e_{2}^{2}$, such that $(\alpha / \beta) e_{1}^{1} \leq e_{2}^{2}$. The specific circumstance in this example that contributes to fulfillment of the condition is essentially the same as in the previous example.

\section{Proofs}

Our proofs of Proposition 3.1 and Theorem 3.8 are based on the following two simple observations on strategies in the Bayesian pure exchange economy (Lemmas 5.1 and 5.2). The former is due to Hahn and Yannelis (1997, proposition 6.10, p. 401), and the latter is due to Ichiishi and Radner (1999, lemma 6.3, p. 330).

Lemma 5.1. Let $z: T \rightarrow \mathbf{R}^{l: \# N}$ be a private measurable net-trade plan in a Bayesian pure exchange economy $\mathcal{E}_{p e}$, such that for each $t \in T$, the total net trade is exactly equal to $\mathbf{0}, \sum_{j \in N} z^{j}\left(t^{j}\right)=\mathbf{0}$. Then, each plan $z^{j}$ is a constant function, hence is Bayesian incentive-compatible.

Lemma 5.2. Let $z^{S}, f^{S}: T^{S} \rightarrow \mathbf{R}^{l \cdot \# S}$ be private measurable functions in a Bayesian pure exchange economy $\mathcal{E}_{p e}$, such that $\sum_{j \in S} z^{j}\left(t^{j}\right) \leq \sum_{j \in S} f^{j}\left(t^{j}\right)$ for every $t^{S} \in T^{S}$. Then, there exists a private measurable function $z^{* S}: T^{S} \rightarrow \mathbf{R}^{l \cdot \# S}$, such that $z^{S} \leq z^{* S}$ and $\sum_{j \in S} z^{* j}\left(t^{j}\right)=\sum_{j \in S} f^{j}\left(t^{j}\right)$ for every $t^{S} \in T^{S}$.

In the light of Lemmas 5.1 and 5.2, we can restrict our attention to constant net-trade plans such that for each type profile the total net trade is exactly equal to $\mathbf{0}$. 


\subsection{Proof of Proposition 3.1}

The proof is the same as Wilson's (1978, p. 814) proof of nonemptiness of his coarse core. The only difference is that in our setup, we only use private-measurable net-trade plans as strategies.

Indeed, given $S \in \mathcal{N}$ and $E \in \bigwedge_{j \in S} \mathcal{T}^{j} \backslash\{\emptyset\}$, define the agent-coalition $(S, E)$ by

$$
(S, E):= \begin{cases}\bigcup_{j \in S}\left[\{j\} \times T^{j}\right] & \text { if \#S } \geq 2, \\ \{j\} \times E^{j} & \text { if } S=\{j\}\end{cases}
$$

(here, $E=T$ if \#S $\geq 2$, and $E=E^{j} \times T^{N \backslash\{j\}}, E^{j} \in \mathcal{T}^{j}$, if $S=\{j\}$ ). Define the set of attainable utility allocations for a blocking coalition $(S, E)$,

$$
V(S, E):=\left\{u \in \mathbf{R}^{A} \mid \exists z^{S} \in F^{\prime S}: \forall\left(j, t^{j}\right) \in(S, E): u_{\left(j, t^{j}\right)} \leq E u^{j}\left(z^{j}+e^{j} \mid t^{j}\right) .\right.
$$

Then the non-side-payment game $V$ is balanced, so by Scarf's (1967) theorem, there exists a private-measurable net-trade plan $z^{\prime}$ which gives rise a coarse core utility allocation.

By Lemma 5.2 there exists a private-measurable net-trade plan $z^{*}$, such that $z^{\prime} \leq z^{*}$ and that $\sum_{j \in N} z^{*}(t)=\mathbf{0}$ for every $t \in T$. By Lemma 5.1 the plan $z^{*}$ is Bayesian incentive-compatible. By the weak monotonicity of each $E u^{j}\left(\cdot \mid t^{j}\right), z^{*}$ is the required Bayesian incentive-compatible coarse core net-trade plan.

\subsection{Proof of Emptiness of the Bayesian Incentive-Compatible Interim Core in} Example 3.4

By abuse of notation, when we have a constant plan $z^{j}$, we also use the notation $z^{j}$ for the image of the function. The same abuse of notation for $e^{j}$. Let $z^{1}$ be a constant function, such that $z^{1} \geq-e^{1}$. Then,

$$
u^{1}\left(z^{1}+e^{1}, t\right)= \begin{cases}z_{2}^{1} & \text { if } t=l \\ 10\left(1+z_{1}^{1}\right)+z_{2}^{1} & \text { if } t=h .\end{cases}
$$

For a constant function $z^{2}$ for which $z^{2} \geq-e^{2}$,

$$
E u^{2}\left(z^{2}+e^{2}\right)=7.5 z_{1}^{2}+z_{2}^{2}+w .
$$

Lemmas 5.1 and 5.2 guarantee that we can consider only constant plans.

In the grand coalition $N$, consumer 1's (constant) strategy has the range

$$
\left(\begin{array}{c}
-1 \\
0
\end{array}\right) \leq z^{1} \leq\left(\begin{array}{l}
0 \\
w
\end{array}\right)
$$

and consumer 2's strategy is given by $z^{2}=-z^{1}$.

We consider the individual rationality, viz., the conditions on a constant strategy bundle $z \in F^{\prime N}$ for no singleton coalition to interim block. Coalition $\{1\}$ cannot block at $t=l$, iff

$$
z_{2}^{1} \geq 0 .
$$


Coalition $\{1\}$ cannot block at $t=h$, iff

$$
10\left(1+z_{1}^{1}\right)+z_{2}^{1} \geq 10
$$

Coalition $\{2\}$ cannot block, iff

$$
-7.5 z_{1}^{1}-z_{2}^{1}+w \geq w
$$

The only $z^{1}$ which satisfies (7)-(9) is the no-trade: $z^{* 1}=\mathbf{0}$. This is, therefore, the only possible interim core strategy.

We will show that the no-trade $z^{* 1}$ is blocked by $N$ at $t=l$, which will complete the proof that this specific model has an empty core. (The no-trade cannot be blocked by $N$ at $t=h$, but this fact is immaterial.) Let $z \in F^{\prime N}$ be a constant strategy proposed in order to block $z^{* 1}$. Consumer 2 agrees to the blocking, iff

$$
-7.5 z_{1}^{1}-z_{2}^{1}+w>w \text {. }
$$

Consumer 1 agrees to the blocking at $t=l$, iff

$$
z_{2}^{1}>0
$$

Both agree to the blocking at $t=l$ iff (10) and (11) are satisfied. There are many $z^{1}$ satisfying these two inequalities, e.g.,

$$
z^{1}=\left(\begin{array}{c}
-1 \\
6
\end{array}\right)
$$

We turn to the proof of Theorem 3.8. We actually establish conditions for the existence of a Bayesian incentive-compatible interim core net-trade plan that is stronger than the one given in Definition 2.3, in that it is stable even against a defecting coalition within which (interim) utilities are freely transferred.

\subsection{Proof of Theorem 3.8}

A constant strategy bundle $t \mapsto c^{*}$ is feasible in the grand coalition (satisfies condition (i) of Definition 2.3), if

$$
\begin{aligned}
& \forall j \in N: c^{* j} \geq-\underline{e}^{j}, \\
& -\sum_{i \in N} c^{* i} \geq \mathbf{0} .
\end{aligned}
$$

$\left(\right.$ Recall $\underline{e}^{j}:=\inf _{t^{j} \in T^{j}} e^{j}\left(t^{j}\right)$.) It is coalitionally stable (satisfies condition (ii) of Definition 2.3), if

$$
\forall B \in \mathcal{B}_{0}: \sum_{j \in S(B)} a^{j}\left(t^{j}(B)\right) c^{* j} \geq v(B) .
$$

A constant map $T \rightarrow\left(\mathbf{R}^{l}\right)^{N}, t \mapsto c^{*}$ is the required Bayesian incentive-compatible interim core net-trade plan, if $c^{*}$ satisfies the above linear inequality system (12)-(14). By a version ${ }^{5}$

\footnotetext{
${ }^{5}$ We use the following version: Let $A$ be an $m \times n$ matrix, and let $b$ be a $m \times 1$ matrix. Then, there exists $x \in \mathbf{R}^{n}$, such that $A x \geq b$, iff for every $1 \times m$ matrix $\lambda \geq \mathbf{0}$ for which $\lambda A=\mathbf{0}$ it follows that $\lambda b \leq 0$.
} 
of the Minkowski-Farkas lemma, we can obtain a necessary and sufficient condition for the existence of such $c^{*}$. In the following, $\lambda:=\left\{\lambda_{B}\right\}_{B \in \mathcal{B}_{0}}, \mu:=\left\{\mu^{j}\right\}_{j \in N}$, and $v$ are $\# \mathcal{B}_{0}$-dimensional, $(\# N) l$-dimensional, and $l$-dimensional row vectors, respectively (each $\mu^{j}$ is an $l$-dimensional subvector). The linear inequality system (12)-(14) has a solution, iff

$$
\begin{aligned}
& \left(\forall(\lambda, \mu, v) \in \mathbf{R}_{+}^{\left(\# \mathcal{B}_{0}\right)+(\# N) l+l}: \sum_{B \in \mathcal{B}_{0}: S(B) \ni j} \lambda_{B} a^{j}\left(t^{j}(B)\right)+\mu^{j}-v=\mathbf{0} \text { for all } j \in N\right): \\
& \sum_{B \in \mathcal{B}_{0}} \lambda_{B} v(B)-\sum_{j \in N} \mu^{j} \underline{e}^{j}-v \mathbf{0} \leq 0 .
\end{aligned}
$$

By eliminating $v$, and by observing that $a^{j}\left(t^{j}(B)\right), \lambda_{B}$ and $\mu^{j}$ are nonnegative, we obtain the condition of Theorem 3.6.

\section{Information-revelation through coalition formation}

Consumer $j$ 's very act of joining a coalition may reveal (a part of) his private information, so condition (ii) of Definition 2.3 (coalitional stability condition for a Bayesian incentive-compatible interim core net-trade plan) may be inappropriate. This observation has been known among the researchers of the present research area as a folklore (see, e.g., Ichiishi and Yamazaki (2002, Subsection 3.2.2)).

The following proposition clarifies a rather specific case in which such information revelation does not influence the consumers' final decision on coalition formation; essentially, it is the situation in which information on the other consumers' types $T^{S \backslash\{j\}}$ is irrelevant to j's interim expected utility.

Proposition 6.1. Let $j \in S$. Assume the $\mathcal{T}^{j}$-measurability of $j$ 's strategies. Assume also the no-externality, that is, $u^{j}$ depends only on $\left(c^{j}, t^{j}\right) \in \mathbf{R}_{+}^{l} \times T^{j}$. Then, revelation of information on $T^{S \backslash\{j\}}$ does not affect $j$ 's decision in regard to joining coalition $S$.

Proof. Let $z^{S} \in F^{\prime S}$. By the no externality,

$$
E u^{j}\left(z^{j}+e^{j} \mid t^{j}\right)=u^{j}\left(z^{j}\left(t^{j}\right)+e^{j}\left(t^{j}\right), t^{j}\right) .
$$

Suppose that willingness of the members $S \backslash\{j\}$ to choose $z^{S}$ reveals information $A$ ( $\subset$ $\left.T^{S \backslash\{j\}}\right)$. Then,

$$
E u^{j}\left(z^{j}+e^{j} \mid t^{j}, A\right)=u^{j}\left(z^{j}\left(t^{j}\right)+e^{j}\left(t^{j}\right), t^{j}\right) .
$$

So, consumer $j$ 's decision in regard to whether or not to accept $z^{S}$ instead of any other plan $z^{\dagger S}$ does not change after obtaining information $A$. 


\section{Acknowledgements}

We express our thanks to Rajiv Vohra; the discussions with him prompted our research reported in this paper. Thanks are also due to an anonymous referee for his/her suggestions.

\section{References}

d'Aspremont, C., Gérard-Varet, L.-A., 1979. Incentives and incomplete information. Journal of Public Economics 11, 22-45.

Hahn, G., Yannelis, N.C., 1979. Efficiency and incentive compatibility in differential information economies. Economic Theory 10, 383-411.

Harsanyi, J.C., 1967/1968. Games with incomplete information played by 'Bayesian' players, Management Science: Theory 14, 159-182 (Part I), 320-334 (Part II), 486-502 (Part III).

Ichiishi, T., Idzik, A., 1996. Bayesian cooperative choice of strategies. International Journal of Game Theory 25, 455-473.

Ichiishi, T., Radner, R., 1999. A profit-center game with incomplete information. Review of Economic Design 4, 307-343.

Ichiishi, T., Yamazaki, A., 2002. Preliminary results for cooperative extensions of the Bayesian game, Discussion Paper \#2001-9, Graduate School of Economics, Hitotsubashi University.

Radner, R., 1968. Competitive equilibrium under uncertainty. Econometrica 36, 31-58.

Scarf, H., 1967. The core of an $N$-person game. Econometrica 35, 50-69.

Vohra, R., 1999. Incomplete information, incentive compatibility, and the core. Journal of Economic Theory 86, 123-147.

Wilson, R., 1978. Information efficiency, and the core of an economy. Econometrica 46, 807-816.

Yannelis, N.C., 1991. The core of an economy with differential information. Economic Theory 1, 183-198. 